Corrigendum

\title{
Corrigendum to "Sodium Tanshinone IIA Sulfonate Attenuates Erectile Dysfunction in Rats with Hyperlipidemia"
}

\author{
Liren Zhong, ${ }^{1}$ Wei Ding, ${ }^{1,2}$ Qinyu Zeng, ${ }^{1}$ Binglin He, ${ }^{1}$ Haibo Zhang, ${ }^{1}$ Li Wang, \\ Junhong Fan, ${ }^{2}$ Shuhua He, ${ }^{1}$ Yuanyuan Zhang $\left(1{ }^{3},{ }^{3}\right.$ and Anyang Wei $\left({ }^{1}{ }^{1}\right.$ \\ ${ }^{1}$ Department of Urology, Nanfang Hospital, Southern Medical University, Guangzhou, Guangdong, China \\ ${ }^{2}$ Department of Urology, The First Affiliated Hospital of Guizhou University of Traditional Chinese Medicine, Guiyang, \\ Guizhou, China \\ ${ }^{3}$ Wake Forest Institute of Regenerative Medicine University, Wake Forest School of Medicine, Winston-Salem, North Carolina, USA \\ Correspondence should be addressed to Yuanyuan Zhang; yzhang@wakehealth.edu and Anyang Wei; profwei@126.com \\ Received 11 October 2020; Accepted 11 October 2020; Published 16 October 2020 \\ Copyright (C) 2020 Liren Zhong et al. This is an open access article distributed under the Creative Commons Attribution License, \\ which permits unrestricted use, distribution, and reproduction in any medium, provided the original work is properly cited.
}

In the article titled "Sodium Tanshinone IIA Sulfonate Attenuates Erectile Dysfunction in Rats with Hyperlipidemia" [1], there was a spelling error in author Qinyu Zeng's name in the author list, where "Qingyu Zeng" should have read "Qinyu Zeng." This is corrected as shown above.

\section{References}

[1] L. Zhong, W. Ding, Q. Zeng et al., "Sodium tanshinone IIA sulfonate attenuates erectile dysfunction in rats with hyperlipidemia," Oxidative Medicine and Cellular Longevity, vol. 2020, Article ID 7286958, 13 pages, 2020. 